\section{Superconducting ceramics}

\section{Solitons and superconductivity}

\section{Alan Bishop}

THE discovery of the new ceramic superconductors, whose high transition temperatures cannot easily be explained by traditional ('BSC') theories of superconductivity, has provoked many theoreticians to dust off and remould exotic theories originally intended for other circumstances. Even though the contributions are coming from very different fields of physics, that of T. D. Lee (who is best known for his theory of parity violation in nuclear disintegration) on page 460 of this issue $^{1}$ may seem more surprising than most. He shows that several theories invoke a single mathematical concept the soliton ${ }^{2}$ - that itself has appeared in many diverse disciplines.

In a strict mathematical sense, solitons are solutions of a limited subset of nonlinear partial differential equations in one space dimension and time. Examples are the sine-Gordon, nonlinear Schrödinger and Korteweg-de Vries equations, approximately describing phenomena in hydrodynamics, optics, plasmas and much more $^{2}$. The most familiar solitons have spatially limited kink, pulse or envelope shapes (see figure). Solitons interact with each other completely nondestructively, suffering only phase-shifts during collisions. This ideal particle-like characteristic is one of many remarkable properties accompanying the integrable structure of the underlying equations.

The notion of a soliton has been widely generalized in the past decade, especially by physicists in condensed-matter and field theory. In practical usage ${ }^{2}$, it now signifies a particle-like lump - a spatially localized, finite-energy collective structure that may or may not propagate and that need not suffer purely elastic collisions with other solitons. In this generalized sense, soliton ('defect') classification in higher spatial dimensions by topological arguments has been valuable, such as in nonlinear elastic media, superfluid helium and field theory (where solitons have been advocated as the means to unify particles and field equations).

A similar situation prevails in discussions of quantum solitons ${ }^{2}$. Quantization of any soliton is possible in principle but exact procedures are available for strict solitons only. In fact, quantizing strict solitons is precisely equivalent to solving 'exactly solvable' problems in onedimensional quantum many-body physics or field theories, or two-dimensional classical statistical mechanics.

An important example of a generalized soliton in condensed matter ${ }^{2}$ is the polaron, often referred to as a 'bag' in equivalent field-theoretical problems. Coupling between two or more fields results in a self-consistent feedback that becomes stabilized as a localized deformation in the fields. For example, coupling between electrons (or their absences, termed holes) and phonons can result in localization of an electron in a localized deformation of the phonon field, that is of the associated atomic lattice - the elastic energy lost in deforming the lattice is compensated by a gain in electronic energy. Describing the structure and dynamics of polarons has exercised the best theoreticians for several decades as a fundamental problem in many-body physics ${ }^{3}$. But these objects have enjoyed a revival with the

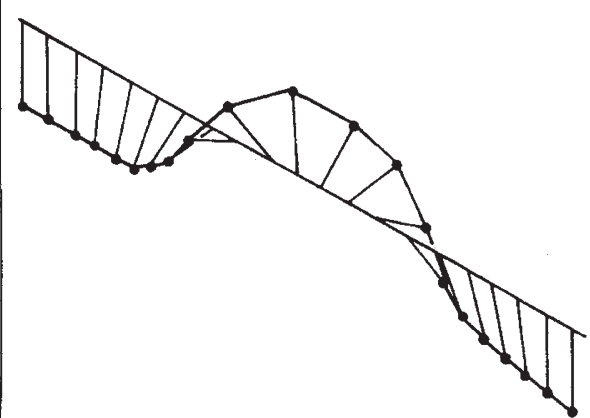

A mechanical model of a soliton. Rods can swing around a rigid bar and are connected by elastic string. A twist, once introduced, cannot be removed, and keeps its shape because of tension in the string. (Courtesy of Ian Stewart and Oxford University Press.)

advent of quasi-one- and two-dimensional solid-state materials because they are stable over larger parameter ranges in these reduced dimensions.

There are several important characteristics of the remarkable new hightemperature superconductors. First, they are ceramics, not metals, so it is not surprising that they display microtexture on many scales. Second, it seems that the active components are planes (or possibly chains) of copper and oxygen atoms. The reduced dimensionality enhances competition between different modes of collective ground-state ordering of spins and/ or charges of the copper ions. Such competitions can be described within familiar model hamiltonians (such as Hubbard models) but the interplay of lowdimensionality and the coupling strengths of electron-electron and electron-phonon interactions forces us to address unfamiliar regimes of those models. Third, excitations created by changing the stoichiometry or the doping (counter) ions (which result in adding electrons or holes in the ground state) are similarly rich, depending on the reference ground state as well as the electron-electron and electronphonon interactions.

In this setting, solitons are becoming a recurrent theme in the rapidly evolving theoretical literature. First, microstructures observed by transmission electron microscopy show 'twinning' textures 'solitonic' striped phase lattices of dislocations (with a spacing of several hundred Angstroms) separating variants of the low-temperature lattice structure. Such microstructure can affect the mechanical strength of the materials. Also, it is hotly debated ${ }^{4,5}$ whether the microstructure can substantially raise the transition temperature of the superconducting phases. Second, the excitations referred to above in simple many-body hamiltonians are typically polaron-like localized states. Depending on whether it is purported that the superconductivity is produced predominantly by spin fluctuations, charge fluctuations or both, theories lead to electron (or hole) polarons, spin-polarons (spin fluctuations localized in a lattice deformation) or similar 'spin-bags'. A popular notion is then that these polarons may bind in pairs (bipolarons) which can behave as 'bosons' and undergo Bose condensation into a macroscopic superconducting coherent quantum state. Relationships between real space pairing and the usual momentum space pairing of the conventional BCS theory of superconductivity also arise in polaron-based descriptions of strong-coupling BCS.

Whether any of the above soliton mechanisms are relevant in the new materials is still not clear. Indeed it is quite possible that different mechanisms will operate as we learn to tune the synthesis of the materials through a more general class. T. D. Lee's contribution in this issue emphasizes from a field-theoretical perspective the polaron nature of many of the proposed many-body approaches. $\mathrm{He}$ observes that the various theories typically lead to equations studied elsewhere (often as model field theories) and known to have soliton 'bag' solutions. A central feature of soliton equations is indeed their genericity - the same equation can arise in very different physical contexts because of their common underlying ingredients. With so many new researchers attracted by the challenge of high-temperature superconductors, it is important that the lesson from soliton studies of crossreferencing between different disciplines is not lost.

\footnotetext{
1. Lee, T.D. Nature 330, 460-461 (1987)

. Bishop, A.R et al. Nonlinearity in Condensed Matter (Springer, Heidelberg, 1987)

(Springer, Heidelberg, 1987).

3. Emin, D. Physics Today 34 (June 1982).
4. Horovitz, B., Krumhansl, J.A. \& Barsch, G. Phys. Rev. B. (in the press).

5. Mueller, F. et al. Phys. Rev. Lett. (in the press)
}

Alan Bishop is in the Theoretical Division and the Center for Nonlinear Studies, Los Alamos National Laboratory, Los Alamos, New Mexico 87545, USA. 